\title{
Doppler de arteria umbilical en fetos con síndrome de Down
}

\section{Umbilical artery Doppler in fetuses with Down syndrome}

\section{Juan C. Bustos-Vidal* y Álvaro Herrera-Alcaíno}

Servicio y Departamento de Obstetricia y Ginecología, Unidad de Medicina Materno Fetal, Universidad de Chile, Hospital San Juan de Dios, Santiago, Chile

\section{Resumen}

Introducción: La aneuploidía más común entre los recién nacidos vivos es el síndrome de Down (SD). En estos niños el crecimiento está disminuido, con una frecuencia del $25 \%$ de restricción del crecimiento intrauterino, pero no se ha establecido el papel de la insuficiencia placentaria. El objetivo es estudiar la resistencia placentaria a través del Doppler de arteria umbilical con índice de pulsatilidad (IP) y el tiempo medio de desaceleración (t/2), y el posible efecto de la insuficiencia placentaria en fetos con SD. Método: Se realizó Doppler en la arteria umbilical en 78 fetos con SD, se midieron el IP y el t/2, y se compararon los resultados con los pesos de nacimiento. Resultados: Se estudiaron 78 fetos con SD con 214 mediciones Doppler. El t/2 y el IP estaban alterados en el 71,5\% y el 65\% de las mediciones, respectivamente. La incidencia de $t / 2$ alterado aumenta con la edad gestacional desde un $28,6 \%$ a las $15-20$ semanas hasta un $89,3 \%$ sobre las 36 semanas ( $p<0,01)$; cifras similares se observan para el IP. La clasificación de los pesos fue: $64 \%$ adecuados, $12 \%$ grandes $y$ $24 \%$ pequeños para la edad gestacional. La última medición de $t / 2$ antes del parto era normal en el $17 \%$ y estaba alterada en el $83 \%$. En el caso del IP, los valores fueron normales en el $27 \%$ y anormales en el $73 \%$. El peso de nacimiento, la edad gestacional y el porcentaje de niños adecuados para la edad gestacional eran significativamente mayores en el grupo con Doppler normal que en el grupo con Doppler alterado. El z-score del t/2 estaba marcadamente alterado (-2.23), pero el del peso de nacimiento solo estaba algo disminuido (-0,39). La mortalidad perinatal fue del $10 \%$, significativamente mayor cuando el flujo diastólico era ausente o reverso. Conclusiones: El estudio demuestra que los fetos con SD tienen una alta incidencia de alteración del Doppler umbilical para el IP y el t/2, lo cual sugiere una insuficiencia placentaria grave. Este deterioro parece iniciarse hacia el final del segundo trimestre y aumenta con la edad gestacional. Sin embargo, en estos fetos, la insuficiencia placentaria produce una ligera caída en el crecimiento fetal. Como hipótesis general pensamos que en los fetos con SD hay datos claros de insuficiencia placentaria, pero habría algún factor que les protegería de una restricción grave del crecimiento.

Palabras clave: Doppler umbilical. Índice de pulsatilidad. Tiempo medio de desaceleración. Síndrome de Down. Insuficiencia placentaria. Restricción del crecimiento fetal.

\section{Abstract}

Introduction: The most common aneuploidy in live newborns is Down syndrome (DS), in these children growth is decreased, with a frequency of 25-36\% of fetal growth restriction (FGR); however, it is not established the role of placental insufficiency. The objective is to study the Doppler of the umbilical artery with pulsatility index (PI) and half peak systolic velocity (hPSV) deceleration time and the possible role of placental insufficiency in fetuses with DS. Method: Doppler was performed in fetuses with DS, the umbilical artery and IP and hPSV were measured, and the results were compared with birth weights.

Disponible en internet: 02-08-2021 Rev Chil Obstet Ginecol. 2021;86(3):258-264 www. rechog.com 
Results: 78 fetuses with DS were studied with 214 Doppler measurements. hPSV and the IP were altered in $71.5 \%$ and $65 \%$ of the measurements; the incidence of abnormal hPSV increases with gestational age from $28.6 \%$ between 15 to 20 weeks, to $89.3 \%$ over 36 weeks $(p<0.01)$, similar figures are observed with respect to the PI. The weight classification was: $24 \%$ of FGR, $12 \%$ of great for age and $64 \%$ of adequate for gestational age (AGA). The last measurement of $h P S V$ before delivery was normal in $17 \%$ of the fetuses and was abnormal in $83 \%$, in the case of PI the normal and abnormal values were 27 and $73 \%$, respectively. Birth weight, gestational age, and the percentage of AGA children were significantly higher in the normal Doppler group than in the abnormal Doppler group. The hPSV z-score was markedly altered (-2.23), but the birth weight $z$-score is slightly decreased (-0.39). Perinatal mortality is $10 \%$ and is significantly higher when diastolic flow is absent or reverse. Conclusions: The study shows that DS fetuses have a high incidence of abnormal umbilical Doppler measured with IP and hPSV, which suggests severe placental insufficiency, this deterioration seems to start towards the end of the second trimester and increases with gestational age. However, in these fetuses, placental insufficiency causes a discrete drop in fetal growth. As a general hypothesis, we think that there is clear evidence of placental insufficiency in fetuses with DS, but there would be some factor that would protect these fetuses from severe growth restriction.

Key words: Umbilical Doppler. Pulsatility index. Half peak systolic velocity deceleration time. Down syndrome. Placental insufficiency. Fetal growth restriction.

\section{Introducción}

La aneuploidía más común entre los recién nacidos vivos es la trisomía 21 o síndrome de Down (SD) ${ }^{1,2}$, con una frecuencia general de 1:600. Hasta hace tres décadas, la única manera de realizar la detección prenatal era considerar la edad de la paciente como factor de riesgo o el hallazgo de malformaciones sospechosas durante el segundo y el tercer trimestres. La aparición del cribado del primer trimestre, tanto bioquímico como por ultrasonido, de 11-14 semanas (hueso nasal, translucencia retronucal, conducto venoso, insuficiencia tricúspidea y ángulo frontonasal), la introducción de la biopsia de vellosidad corial y la amniocentesis genética, y el desarrollo de las pruebas de FISH y de DNA libre fetal, han permitido el diagnóstico prenatal en el primer trimestre de un gran porcentaje de fetos con aneuploidías ${ }^{3}$.

Durante el segundo y el tercer trimestres se han descrito numerosos signos sugerentes de SD: pielectasia, pie en sandalia, cardiopatía congénita, arteria subclavia derecha anómala, atresia duodenal, braquicefalia, ventriculomegalia, hipoplasia del hueso nasal, edema prenasal, edema retronucal, huesos largos cortos, intestino hiperecogénico y macroglosia ${ }^{4}$; sin embargo, solo recientemente se han descrito alteraciones en el Doppler de la arteria umbilical en los fetos con $\mathrm{SD}^{1,5}$.

En los fetos con SD, los pesos de nacimiento son menores que en los fetos euploides (en promedio, en el percentil 10-25) y también es más frecuente la restricción del crecimiento intrauterino (RCIU), con incidencias del $25-36 \%$. La causa de esta alteración de crecimiento ha sido atribuida a «origen fetal» 0 «bajo potencial de crecimiento", terminología vaga que no es apoyada por datos sólidos; una segunda explicación sería la insuficiencia placentaria'.

Nuestro grupo de trabajo ha descrito un índice de conductancia placentaria, el tiempo medio de desaceleración (t/2) (en inglés half peak systolic velocity deceleration time [hPSV]), con un nomograma de 877 fetos $^{6}$, que demuestra ser similar o mejor que el índice de pulsatilidad (IP) para el diagnóstico de insuficiencia placentaria en fetos euploides con RCIU? Ya hemos publicado una serie de Doppler umbilical en fetos con $\mathrm{SD}^{8}$, y en este artículo extendemos ampliamente esta casuística aumentando el número de fetos un 30\% y el número de estudios Doppler un $44 \%$.

El objetivo de nuestro estudio es analizar la resistencia placentaria a través del Doppler umbilical y el posible papel de la insuficiencia vasculoplacentaria en la etiología del RCIU en fetos con SD.

\section{Método}

Este es un estudio ambispectivo con datos retrospectivos y longitudinales, realizado en pacientes del servicio de obstetricia y ginecología del Hospital San Juan de Dios, de la Universidad de Chile, en Santiago de Chile. Se revisaron las bases de datos entre 2004 y 2020 en busca de fetos con SD cuyo diagnóstico se realizó en la etapa prenatal (por cariograma o fuerte sospecha ecográfica) o posnatal. Todos los fetos tenían cariograma prenatal o posnatal, con datos de parto, peso y edad gestacional conocidos. Los embarazos hasta 2017 ya habían sido presentados en la serie anterior $^{3}$. El seguimiento en los fetos con diagnóstico prenatal de SD se realiza con control clínico, ecografía 
y Doppler seriado cada 3-4 semanas; si además se detecta RCIU, el control ecográfico es semanal o bisemanal, en forma análoga a los fetos euploides.

En estos fetos se realizó la medición de Doppler en la arteria umbilical según las normas de la International Society of Ultrasound in Obstetrics and Gynecology para fetos en apnea, con un mínimo de tres ondas similares. Se midieron el IP y el t/2, y las mediciones se clasificaron según la edad gestacional en cinco grupos: menos de 20 semanas, $20+0$ a $24+6$ semanas, $25+0$ a 29+6 semanas, $30+0$ a 34+6 semanas y más de 35 semanas. Se excluyeron para el análisis aquellas mediciones con un intervalo menor de 7 días. Se consideró un IP alterado o anormal cualquier valor sobre el percentil 95 de las tablas de Acharya, et al. ${ }^{9}$ en fetos sobre 19 semanas, o de Bustos y González $^{10}$ en fetos bajo 19 semanas. En el caso del t/2, se consideraron alterados 0 anormales los valores bajo el percentil $5^{10}$.

Los recién nacidos se catalogaron como RCIU grave (bajo el percentil 3), RCIU moderado (entre los percentiles 3 y 10), adecuado para la edad gestacional (percentiles 10-90) o grande para la edad gestacional, según la tabla nacional de Alarcón-Pittaluga ${ }^{11}$. Los pesos de nacimiento se compararon con la última medición de Doppler previa al parto. Para este análisis se descartaron cinco fetos con hidrops, ascitis o hidrotórax; por lo tanto, se estudiaron 73 recién nacidos con peso seco.

Para el análisis estadístico se utilizaron las pruebas ji al cuadrado, Fisher y $t$ de Student. Para los recién nacidos se calcularon el z-score del peso y el z-score del $t / 2$.

\section{Resultados}

Se identificaron 78 fetos con SD que cumplían con los criterios de inclusión. El diagnóstico fue prenatal en 66 casos (85\%), de los cuales $36(46 \%)$ se confirmaron con cariograma o FISH prenatal y $30(38 \%)$ tenían fuerte sospecha ecográfica. En 12 casos (15\%) no hubo diagnóstico prenatal y fue un hallazgo al nacimiento. En estos fetos se realizaron 214 mediciones de Doppler, con un rango de 12 a 39 semanas (por cada feto: rango 1-8, mediana 2).

Los resultados del IP y del t/2 del Doppler en la arteria umbilical se muestran en la tabla 1 y en las figuras 1 y 2 . En el global de todas las mediciones, el $\mathrm{t} / 2$ y el IP eran anormales en el $71,5 \%$ y el $65 \%$, respectivamente. Según los rangos de edad gestacional, se observa que el número de exámenes con t/2 anormal aumenta con la edad gestacional desde un $28,6 \%$ en el grupo de fetos antes de las 20 semanas hasta
Tabla 1. Índice de pulsatilidad y tiempo medio de desaceleración según la edad gestacional en fetos con síndrome de Down

\begin{tabular}{|l|c|c|c|c|c|c|}
\hline $\begin{array}{l}\text { Edad } \\
\text { gestacional }\end{array}$ & \multicolumn{2}{|c|}{ t/2 alterado } & $\begin{array}{c}\text { IP } \\
\text { alterado }\end{array}$ & & \\
\hline (semanas) & $n$ & $\%$ & $n$ & AR & $\%$ & Total \\
\hline $12-20$ & 8 & 28,6 & 10 & 3 & 35,7 & 28 \\
\hline $21-25$ & 18 & 62,1 & 14 & 0 & 48,3 & 29 \\
\hline $26-30$ & 42 & 76,4 & 38 & 2 & 69,1 & 55 \\
\hline $31-35$ & 60 & 81,1 & 54 & 6 & 73,0 & 74 \\
\hline $36-40$ & 25 & 89,3 & 23 & 0 & 82,1 & 28 \\
\hline Total & 153 & $71,5 \%$ & 139 & 11 & $65 \%$ & 214 \\
\hline
\end{tabular}

AR: flujo diastólico ausente o reverso; IP: índice de pulsatilidad; t/2: tiempo medio de desaceleración.

$\mathrm{t} / 2$ alterado: $<\mathrm{p} 5$.

IP alterado: $>$ p95.

${ }^{*} p<0,01$ (prueba de ji al cuadrado)

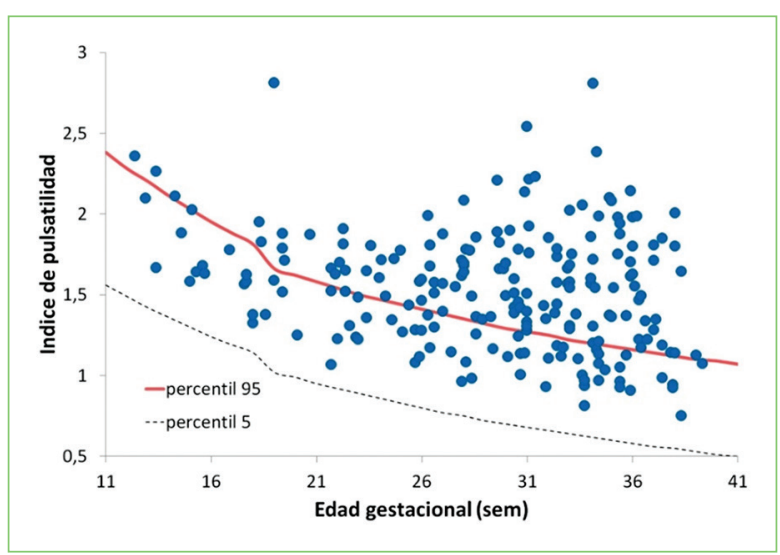

Figura 1. Índice de pulsatilidad en fetos con síndrome de Down.

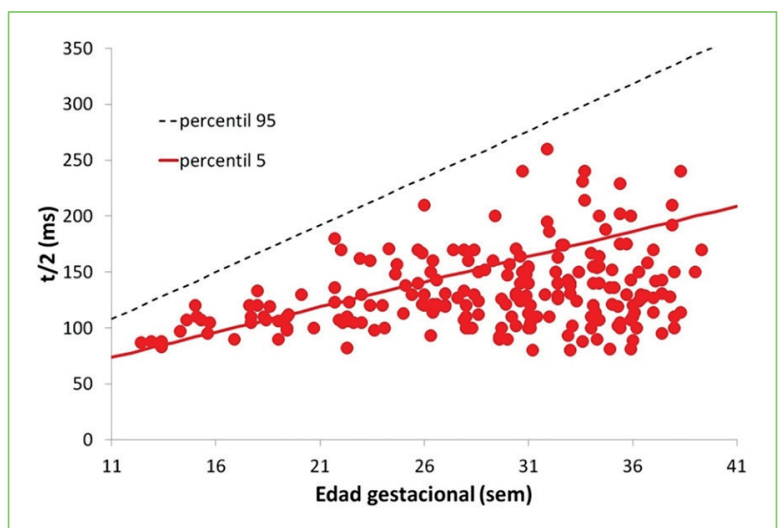

Figura 2. Tiempo medio de desaceleración ( $t / 2$ ) en milisegundos en fetos con síndrome de Down. 
Tabla 2. Índice de pulsatilidad y tiempo medio de desaceleración según la clasificación de peso de nacimiento en fetos con síndrome de Down

\begin{tabular}{|c|c|c|c|c|c|c|}
\hline & \multicolumn{2}{|c|}{ IP normal } & \multicolumn{2}{|c|}{ IP alterado } & \multicolumn{2}{|c|}{ Total } \\
\hline & n & $\%$ & n & $\%$ & n & $\%$ \\
\hline GEG* & 2 & $22,2 \%$ & 7 & $77,8 \%$ & 9 & $11,5 \%$ \\
\hline AEG & 18 & $36 \%$ & 32 & $64 \%$ & 50 & $64,1 \%$ \\
\hline PEG moderado & 0 & $0 \%$ & 14 & $100 \%$ & 14 & $17,9 \%$ \\
\hline PEG grave & 1 & $20 \%$ & 4 & $80 \%$ & 5 & $6,4 \%$ \\
\hline \multirow[t]{2}{*}{ Total } & 21 & & 57 & & 78 & \\
\hline & t/2 normal & & t/2 alterado & & n & $\%$ \\
\hline GEG* & 1 & $11,1 \%$ & 8 & $88,9 \%$ & 9 & $11,5 \%$ \\
\hline AEG & 12 & $24 \%$ & 38 & $76 \%$ & 50 & $64,1 \%$ \\
\hline PEG moderado & 0 & $0 \%$ & 14 & $100 \%$ & 14 & $17,9 \%$ \\
\hline PEG grave & 0 & $0 \%$ & 5 & $100 \%$ & 5 & $6,4 \%$ \\
\hline Total & 13 & & 65 & & 78 & \\
\hline
\end{tabular}

un $89,3 \%$ en el grupo sobre 36 semanas ( $p<0,01$ ). Una tendencia similar se observa respecto al IP: un $35,7 \%$ de valores alterados en el grupo de $15-20$ semanas y un $82,1 \%$ sobre las 36 semanas $(p<0,01)$.

La edad gestacional promedio al parto fue de 35,9 semanas (rango: 29-40 semanas). El peso promedio al nacer fue de $2726 \pm 726 \mathrm{~g}$. La clasificación de los pesos se muestra en tabla 2: 19 recién nacidos tenían RCIU (24,4\%), de los cuales en $5(6,4 \%)$ era grave, 50 niños eran adecuados para la edad gestacional $(64,1 \%)$ y $9(11,1 \%)$ eran grandes para la edad (incluyen cinco casos de hidrops, hidrotórax o ascitis). De los niños con $\mathrm{RCIU}$, el $100 \%$ tenían el $\mathrm{t} / 2$ alterado y el $95 \%$ tenían el IP alterado.

Los datos del parto respecto a la última medición de Doppler en los 73 recién nacidos con peso seco se consignan en la tabla 3. Se observa que en el grupo con Doppler normal el peso de nacimiento, la edad gestacional y la frecuencia de peso adecuado para la edad gestacional (AEG) son significativamente mayores en comparación con el grupo de Doppler anormal, ya sea con IP o t/2 (con la excepción de la edad gestacional en $t / 2$ ). Además, cuando el IP es normal, los fetos pesan $3151 \mathrm{~g}$ en promedio, con solo un 5,3\% de RCIU.

Se analizaron los z-score de los pesos de nacimiento frente al $z$-score del $t / 2$. Los fetos con SD tienen un $z$-score promedio de $t / 2$ de $-2,23$, muy alterado, pero el peso de nacimiento solo está moderadamente disminuido, con un z-score de -0,39.

Hubo dos mortinatos y seis mortineonatos, con una mortalidad perinatal del 10,3\% (8/78). En los 8 pacientes cuyo último Doppler umbilical fue flujo cero o reverso hubo una mortalidad perinatal del 37,5\% (3/8), significativamente mayor que en los fetos con flujo diastólico presente, que fue del 7,1\% $(5 / 70)(p<0,05)$.

Se presenta un caso clínico muy ilustrativo: una paciente con 19 semanas en que se detecta higroma quístico y canal atrio ventricular. El Doppler umbilical tenía flujo cero en diástole, se realizó amniocentesis y el cariograma confirmó el SD. Durante el resto del embarazo el Doppler mejoró, pero mantenía un flujo mínimo en diástole. El IP varió entre 1,6 y 2, y el t/2 entre 90 y $120 \mathrm{~ms}$; todos valores muy alterados. Finalmente el parto fue a las 37 semanas, con un recién nacido con un peso adecuado a la edad gestacional de $3430 \mathrm{~g}$, con $\mathrm{SD}$, y en buenas condiciones excepto la cardiopatía.

\section{Discusión}

Nuestro estudio demuestra que los fetos con SD tienen una alta incidencia de alteración del Doppler umbilical medido con IP y t/2, lo cual sugiere insuficiencia 
Tabla 3. Pesos de nacimiento según Doppler umbilical en fetos con síndrome de Down

\begin{tabular}{|l|c|c|c|c|c|c|}
\hline & t/2 normal & t/2 alterado & P & IP normal & IP alterado & P \\
\cline { 1 - 3 } & Promedio (DE) & Promedio (DE) & & Promedio (DE) & Promedio (DE) \\
\hline Peso (g) & $3104(716)$ & $2560(557)$ & $<0,01$ & $3151(423)$ & $2484(722)$ & $<0,01$ \\
\hline Edad gestacional (sem) & $36,7(1,9)$ & $35,6(2,8)$ & 0,11 & $37,4(1,7)$ & $35,3(2,8)$ & $<0,01$ \\
\hline RCIU/PEG & $0 \%(0 / 13)$ & $31,7 \%(19 / 60)$ & $<0,05$ & $5,3 \%(1 / 19)$ & $33,3 \%(18 / 54)$ & $<0,05$ \\
\hline
\end{tabular}

DE: desviación estándar; PEG: pequeño para la edad gestacional; RCIU: restricción del crecimiento intrauterino.

$\mathrm{t} / 2$ alterado: $<\mathrm{p} 5$

IP alterado: > p95

RCIU/PEG: peso nacimiento $<$ p10.

Pruebas t de Student y Fisher.

placentaria. Esta alteración de la resistencia umbilical parece iniciarse hacia el final del segundo trimestre y aumenta con la edad gestacional.

La incidencia de Doppler anormal medido con t/2 o IP en fetos con SD entre las 21 y 25 semanas (56\%) es más alta que la de todos los marcadores descritos para el segundo trimestre (foco ecogénico intracardiaco, ventriculomegalia, aumento del pliegue nucal, intestino ecogénico, pielectasia, húmero corto, fémur corto, arteria subclavia derecha aberrante), excepto el hueso nasal ausente o hipoplásico (59\%), por lo cual la medición del IP y el t/2 debe ser incorporada en esta lista de marcadores sutiles ${ }^{4}$.

Hasta hace poco eran escasos los datos clínicos de Doppler de la arteria umbilical en SD y solo del primer trimestre $^{12-14}$. En 2015, Flöck et al. ${ }^{1}$ publicaron un estudio con una importante casuística de 281 casos de SD: el $42 \%$ de los IP eran anormales, y este porcentaje aumentaba desde el $32 \%$ en los menores de 20 semanas hasta el $64 \%$ en los mayores de 35 semanas. Estas cifras son similares a las nuestras (35\% y $82 \%$, respectivamente). Al estudiar la conductancia con $\mathrm{t} / 2$, la frecuencia de exámenes anormales es aún mayor (29\% y $89 \%$, respectivamente). A diferencia del de Flöck, et al. ${ }^{1}$, nuestro estudio demuestra que el Doppler alterado (medido con IP o t/2) se correlaciona con una mayor incidencia de prematuros, bajo peso al nacer y $\mathrm{RCIU}$, de modo similar a lo que ocurre con los fetos euploides.

En un estudio de Wagner, et al. ${ }^{5}$, también de 2015, en 42 casos de SD a partir de las 33 semanas, se encontró el IP alterado en el $60 \%$ de los fetos AEG y en el $55 \%$ de los fetos con RCIU. En nuestro estudio, al comparar los 59 niños sobre 33 semanas, el IP estaba alterado en el $62 \%$ de los fetos AEG, pero en aquellos con RCIU las cifras son mayores, con un $93 \%$ de IP alterado, aunque sin significación estadística. EI $z$-score promedio de IP en el estudio de Wagner, et al. ${ }^{5}$ era de $-2,7$, y en el nuestro el $z$-score de $t / 2$ era de $-2,23$.

En el estudio de Flöck, et al. ${ }^{1}$ y en el nuestro se observa que el porcentaje de Doppler umbilical anormal aumenta con la edad gestacional, con cifras similares tanto con IP como con $t / 2$, lo que sugiere un deterioro progresivo de la placenta durante el embarazo. Este hecho contrasta con lo observado en fetos euploides, en los que un incremento en el número y en el diámetro de los vasos en las vellosidades terciarias disminuye la impedancia de la arteria umbilical con la edad gestacional. Los hallazgos en fetos con SD se correlacionan con los estudios histopatológicos de placentas de SD que demuestran una larga serie de alteraciones ultraestructurales, tanto en calidad como cantidad, de las vellosidades coriales, y cordón umbilical con hipotorsión ${ }^{15-21}$. Un interesante resumen publicado por Corry, et al. ${ }^{22}$ en 2016, que aplica los nuevos consensos en histopatología, demuestra que las placentas de los fetos con SD tienen mala perfusión vascular fetal en un $48 \%$ y defectos de maduración vellositaria en un $39 \%$.

Respecto al crecimiento fetal, destacamos tres hechos: primero, en la serie total, a pesar de una alta incidencia de Doppler umbilical anormal, solo hay un $24 \%$ de RCIU; segundo, en el grupo de fetos con Doppler anormal, más del $60 \%$ son AEG, e incluso hay fetos grandes para la edad gestacional; y tercero, en el grupo con Doppler umbilical normal, el peso promedio (3151 g) y la frecuencia de RCIU $(5,3 \%)$ son muy similares a los de los fetos euploides. Estos datos contrastan con lo observado en fetos euploides, en los que el hallazgo de un Doppler umbilical alterado es un fuerte marcador de insuficiencia placentaria y se asocia a una alta frecuencia de RCIU? 
Como hipótesis general, Flöck, et al. ${ }^{1}$ y Wagner, et al. ${ }^{5}$ sugieren que el menor peso de los fetos con SD podría deberse a insuficiencia placentaria. Nosotros, ampliando esta hipótesis, planteamos que en los fetos con SD hay alteraciones en la arquitectura placentaria que condicionan una insuficiencia placentaria importante, pero habría algún factor no conocido que protegería al feto de una restricción grave, como ocurre en los fetos euploides; es decir, al contrario de lo tradicionalmente planteado, estos niños tendrían un mayor potencial de crecimiento que los fetos euploides.

Nuestros datos tienen también implicancias clínicas para el manejo de un feto con SD. Así, la presencia de un Doppler umbilical alterado, pero con crecimiento normal o levemente disminuido, es normal para estos fetos, y no debe determinar una interrupción precoz del embarazo ${ }^{5}$. Sin embargo, sí debe plantearse la interrupción si aparece flujo diastólico ausente 0 reverso, que implica una alta mortalidad perinatal. Desde otro punto de vista, en casos de embarazos con Doppler alterado y crecimiento normal o levemente disminuido en pacientes con control deficitario del embarazo, debe alertar de la posibilidad de un SD no diagnosticado.

Entre las fortalezas de este estudio se encuentra que es la serie más grande de Doppler umbilical en fetos con SD publicada en español y en Hispanoamérica, y la segunda casuística mundial (215 mediciones). Asimismo, es la segunda publicación sobre un índice de conductancia ( $t / 2)$ en fetos con SD.

Entre las debilidades del estudio está que nuestros datos no permiten identificar este factor protector de los fetos con SD.

En el futuro se debería tener una mayor casuística, especialmente de mediciones longitudinales de Doppler, para así confirmar la sugerencia de que estos fetos tienen mejor potencial de crecimiento y explorar la posibilidad de mejorar el rendimiento placentario (ácido acetilsalicílico, vasodilatadores), con el fin hipotético de mejorar el pronóstico neurológico de estos niños, y también incorporar la medición del t/2 o del IP como un fuerte marcador de SD en el segundo y el tercer trimestres, en especial cuando el crecimiento es normal.

\section{Conclusiones}

Se demuestra que los fetos con SD tienen una alta incidencia de alteración del Doppler umbilical que se inicia hacia el final del segundo trimestre y aumenta con la edad gestacional, lo cual sugiere insuficiencia placentaria. La aparición de flujo diastólico ausente o reverso es muy ominosa. Estos hallazgos pueden contribuir a proteger a estos niños de muerte fetal o de un mayor daño neurológico.

\section{Agradecimientos}

Al Dr. Pablo Salinas Carrasco por algunas ideas iluminadoras.

\section{Financiación}

Los autores declaran que el estudio no requirió financiación.

\section{Conflicto de intereses}

Los autores declaran que no tienen ningún conflicto de intereses.

\section{Responsabilidades éticas}

Protección de personas y animales. Los autores declaran que para esta investigación no se han realizado experimentos en seres humanos ni en animales.

Confidencialidad de los datos. Los autores declaran que han seguido los protocolos de su centro de trabajo sobre la publicación de datos de pacientes.

Derecho a la privacidad y consentimiento informado. Los autores han obtenido el consentimiento informado de los pacientes y/o sujetos referidos en el artículo. Este documento obra en poder del autor de correspondencia.

\section{Bibliografía}

1. Flöck A, Remig I, Müller A, Nadal J, Berg C, Gembruch U, et al. Conflicting umbilical artery Doppler findings in fetuses with trisomy 21. Arch Gynecol Obstet. 2015;292:613-7.

2. Bustos JC, Castillo J, Ardiles G. Síndrome de Down: aspectos obstétricos. Rev Chil Obstet Ginecol. 1988;53:224-8.

3. Monni G, Corda V, luculano A, Afshar Y. The decline of amniocentesis and the increase of chorionic villus sampling in modern perinatal medicine. J Perinat Med. 2020 Mar 19. doi: 10.1515/jpm-2020-0035. [Epub ahead of print]

4. Agathokleous M, Chaveeva P, Poon L, Kosinski P, Nicolaides K. Meta-analysis of second-trimester markers for trisomy 21. Ultrasound Obstet Gynecol. 2013;41:247-61.

5. Wagner P, Sonek J, Hoopmann M, Abele H, Brucker S, Kagan KO. Increased umbilical artery pulsatility index in third-trimester fetuses with trisomy 21. Fetal Diagn Ther. 2016;39:100-4.

6. Bustos JC, Paublo M, Ramírez P, Sepúlveda W. Umbilical artery half peak systolic velocity deceleration time throughout pregnancy and its role in fetuses with bradycardia. Ultrasound Obstet Gynecol. 2007;30:952-7.

7. Bustos JC, González V, Sepúlveda W. Umbilical artery half peak systolic velocity deceleration time in fetal growth restriction. Fetal Diagn Ther. 2016;40:128-34.

8. Bustos JC, Herrera A, Sepúlveda W. Umbilical artery pulsatility index and half-peak systolic velocity deceleration time in fetuses with trisomy 21 . J Matern Fetal Neonatal Med. 2020;33:3469-75. 
Rev Chil Obstet Ginecol. 2021;86(3)

9. Acharya G, Wilsgaard T, Berntsen G, Maltau J, Kiserud T. Reference ranges for serial measurements of umbilical artery Doppler indices in the second half of pregnancy. Am J Obstet Gynecol. 2005;192:937-44

10. Bustos JC, González V. Curvas normales de índices de pulsatilidad y tiempo medio de desaceleración de arteria umbilical fetal en población chilena. Rev Chil Obstet Ginecol. 2014;79:255-61.

11. Milad M, Novoa JM, Fabres J, Samamè M, Aspillaga C. Recomendación sobre curvas de crecimiento intrauterino. Rev Chil Pediatr. 2010;81:264-74.

12. Martínez Crespo JM, Comas C, Ojuel H, Puerto B, Borrell A, Fortuny A. Umbilical artery pulsatility index in early pregnancies with chromosome anomalies. Br J Obstet Gynaecol. 1996:103:330-4.

13. Zoppi MA, Ibba RM, Putzolu M, Floris M, Monni G. First trimester umbilical artery pulsatility index in fetuses presenting enlarged nuchal translucency. Prenat Diagn. 2000;20:701-4.

14. Wong $G$, Levine $D$. Fetuses with trisomy 21 having conflicting findings on antenatal testing for fetal well-being. J Ultrasound Med. 2005;24:1541-5

15. Jauniaux E, Hustin J. Chromosomally abnormal early ongoing pregnancies: correlation of ultrasound and placental histological findings. Hum Pathol. 1998;29:1195-9

16. Rochelson B, Kaplan C, Guzmán E, Arato M, Hansen K, Trunca C A quantitative analysis of placental vasculature in the third-trimester fetus with autosomal trisomy. Obstet Gynecol. 1990;75:59-63.
17. Qureshi F, Jacques SM, Johnson MP, Hume RF Jr, Kramer RL, Yaron Y et al. Trisomy 21 placentas: histopathological and immunohistochemical findings using proliferating cell nuclear antigen. Fetal Diagn Ther. 1997;12:210-5.

18. Kuhlmann RS, Werner AL, Abramowicz J, Warsof SL, Arrington J, Levy DL. Placental histology in fetuses between 18- and 23-weeks' gestation with abnormal karyotype. Am J Obstet Gynecol. 1990;163(4 Pt 1): 1264-70.

19. Pidoux G, Gerbaud P, Cocquebert M, Segond N, Badet J, Fournier T, et al. Human trophoblast fusion and differentiation: lessons from trisomy 21 placenta. Placenta. 2012;33 (Suppl):S81-6.

20. Malassine A, Frendo JL, Evain-Brion D. Trisomy 21-affected placentas highlight prerequisite factors for human trophoblast fusion and differentiation. Int J Dev Biol. 2010;54:475-82.

21. Roberts L, Sebire NJ, Fowler D, Nicolaides KH. Histomorphological features of chorionic villi at 10-14 weeks of gestation in trisomic and chromosomally normal pregnancies. Placenta. 2000;21:678-83.

22. Corry E, Mone F, Segurado R, Downey P, McParland P, McAuliffe FM, et al. Placental disease and abnormal umbilical artery Doppler waveforms in trisomy 21 pregnancy: a case-control study. Placenta. 2016; 47:24-8. 